\title{
Inter- and intraspecific sexual discrimination in the flour beetles Tribolium castaneum and Tribolium confusum
}

\author{
JOSÉ M. SERRANO†, LAUREANO CASTRO†, MIGUEL A. TORO*† \\ \& CARLOS LOPEZ-FANJUL: \\ $\dagger$ Departamento de Mejora Genética y Biotecnología, SGIT-INIA, Carretera de La Coruña km 7, 28040 Madrid, Spain \\ and $\ddagger$ Departamento de Genética, Facultad de Ciencias Biológicas, Universidad Complutense, 28040 Madrid, Spain
}

\begin{abstract}
In Tribolium castaneum (CS) and T. confusum (CF), intra- and interspecific rates of homosexual mounting have been measured. The intraspecific results are compatible with the hypothesis of both species being sexually indiscriminate. However, the CF intraspecific rates were very high $(35 \%-53 \%$ of mountings were homosexual), suggesting a lower sexual attractiveness, or a stronger rejection to being mounted, of $\mathrm{CF}$ females relative to conspecific males. CS males discriminate between species but, in interspecific contacts, preferentially mounted CF males rather than CF females. CF males do not discriminate between species, but the loss of sexual attractiveness of $\mathrm{CF}$ females, or their rejection to being mounted, may act as a precopulatory isolation mechanism.
\end{abstract}

Keywords: homosexual mounting, precopulatory isolation mechanisms, Tribolium.

\section{Introduction}

In Tribolium castaneum (CS) and T. confusum (CF), high rates of homosexual mounting have often been reported (see Serrano et al., 1991 for a review). These have been routinely ascribed to 'mistakes', male aggressiveness, female scarcity or crowded laboratory conditions. However, the relative importance of these factors generally has been assumed rather than directly investigated. Thornhill \& Alcock (1983) have proposed an evolutionary interpretation of insect homosexual mounting behaviour. Assuming that the male's main cost of reproduction is the time consumed in discerning the sex of potential mates, increasing the number of mountings per unit of time, at the risk of making some mistakes, may be more advantageous than carefully choosing a partner of the right sex. This implies that the genetic correlation between the rate of homosexual mounting and mating activity must be positive. In laboratory CS populations, a clear direct response to artificial divergent selection for the rate of homosexual mounting was obtained by Castro et al. (1994), indicating the existence of genetic variability for this trait. However, the results did not support Thornhill \& Alcock's hypothesis, as the correlated response for male mating activity was statistically indistinguishable from zero.

*Correspondence. E-mail: toro@inia.es
Serrano et al. (1991) found agreement between the observed rate of homosexual mounting in CS and the one expected by assuming random pair contacts between potential mates, when both sexes equally oppose being mounted. This result has been shown to apply in a variety of experimental situations, indicating that CS males behaved in a totally indiscriminate manner with respect to sex. Intraspecific sexual indiscrimination is more likely to occur in those insect species (such as CS and $\mathrm{CF}$ ) showing a high frequency of homosexual mountings, together with much reduced sensorial perception and lacking both courtship behaviour and obvious external sex differences. Olfaction appears as practically the only means of establishing a relationship among individuals. However, this is attained through a male-secreted pheromone, strongly attractive to both sexes (Faustini et al., 1981; Levinson \& Mori, 1983). Thus, it seemed of interest to enquire if the indiscriminate mounting behaviour of CS was shared by other species of the same genus, such as CF.

The extent to which intraspecific sexual indiscrimination may also imply a certain degree of interspecific indiscrimination has not been investigated. However, the latter could prevent the acquisition of precopulatory reproductive isolation mechanisms between species. Within the genus Tribolium, only postcopulatory prezygotic mechanisms have been reported between those closely related species where viable but sterile hybrids 
can be obtained (CS and T. freemani: Robinson et al., 1994; Wade et al., 1994). Although they show morphological resemblance, CS and CF are phylogenetically quite distant (Juan et al., 1993; Ugarkovic et al., 1996) and $\mathrm{CS} \times \mathrm{CF}$ crosses do not produce viable offspring. Both species can often be found in the same localities but, in laboratory conditions defined by varying temperature and relative humidity, one of them always displaces the other, with high values of these variables favouring CS and low values favouring CF (see Sokoloff, 1974 for a review). In spite of the wide use of CS and CF in competition experiments, only marginal data on interspecific $\mathrm{CS} \times \mathrm{CF}$ mating behaviour have been reported (Taylor \& Sokoloff, 1971; Vardell \& Brower, 1976; Graur \& Wool, 1985). Thus, we have also checked if the lack of intraspecific sexual discrimination can be extended to the interspecific level.

\section{Materials and methods}

\section{Strains and culture conditions}

Two laboratory populations were used: Consejo (CS) and Ramiro (CF). They were both captured near Madrid and have been maintained in cages in our laboratory since 1964 and 1975, respectively, with no artificial selection and minimal inbreeding.

All beetles in this experiment were kept in the same dark incubator at $70 \%$ relative humidity and $33^{\circ} \mathrm{C}$. The culture medium consisted of $95 \%$ whole wheat flour and $5 \%$ dried brewer's yeast by weight, powdered. Pupae were sexed by examination of the genital lobes. Male pupae were always kept individually and female pupae stored at low densities, until tested (12-19 days after adult emergence). Handling was performed at room temperature under constant artificial illumination.

\section{Estimation of rates of homosexual mounting}

Mating cannot be seen within the culture medium because beetles show a strong photonegative reaction.
In this situation, our observations were made as follows. Males were placed in a glass vial $(3 \mathrm{~cm}$ diameter, $3.5 \mathrm{~cm}$ height) with a filter paper floor, together with virgin females, in the numbers specified in Tables 1 and 2 . A white, yellow or blue spot of paint on the thorax randomly identified males and females. For each male, the numbers of homosexual and heterosexual mountings were recorded for a period of $30 \mathrm{~min}$ on each of four consecutive days, the females being substituted by new ones each day. Only those mountings (homo- and heterosexual) in which the mounting male showed symptoms of being sexually aroused (extension of aedagus) were recorded as such. However, heterosexual mountings were not distinguished from intromissions. The rate of homosexual mounting can be calculated for each male as the ratio between the number of homosexual mountings and the total number of mountings recorded (homo- and heterosexual). For each situation, the number of vials observed simultaneously is also indicated in Tables 1 and 2. Our observations indicate that the behaviour of males and females, when being mounted, does not show obvious differences. Active rejection (e.g. aggressive) was not involved, but a passive rejection (noncooperative) was usually observed.

\section{Model}

The following model, proposed by Serrano et al. (1991), provides the framework within which our data can be discussed. It takes into account not only the behaviour of the mounting male but also possible differential reactions of the individuals which are being mounted.

Consider $m$ males and $f=k m$ females within an enclosure, $k$ being the sex ratio among scored individuals. Assume that contacts take place between only two individuals at a time and that they occur at random with respect to sex. Thus, the number of possible contacts will be $\left.\begin{array}{c}m \\ 2\end{array}\right)$ between males and $m f$ between a male and a female. These contacts will result in mounting with probabilities $x$ and $y$, respectively. As females do not

Table 1 Intraspecific mountings in Tribolium castaneum (CS) and T. confusum $(\mathrm{CF})$ : number of males and females tested together in a vial, total number of mountings observed and rate of homosexual mounting $\left(P_{\mathrm{m}}\right)$

\begin{tabular}{lccccccc}
\hline & \multicolumn{2}{l}{ No. males } & & \multicolumn{2}{l}{ No. females } & & \\
\cline { 2 - 4 } \cline { 7 - 8 } No. of vials & CS & CF & & CS & CF & No. mountings & $P_{\mathrm{m}}$ \\
\hline 50 & 0 & 2 & 0 & 2 & 291 & $0.53 \pm 0.03$ \\
30 & 0 & 2 & 0 & 4 & 198 & $0.35 \pm 0.03$ \\
20 & 2 & 0 & 2 & 0 & 456 & $0.32 \pm 0.02$ \\
$20^{*}$ & 2 & 0 & 4 & 0 & 582 & $0.22 \pm 0.02$ \\
\hline
\end{tabular}

*Data from Serrano et al. (1991). 


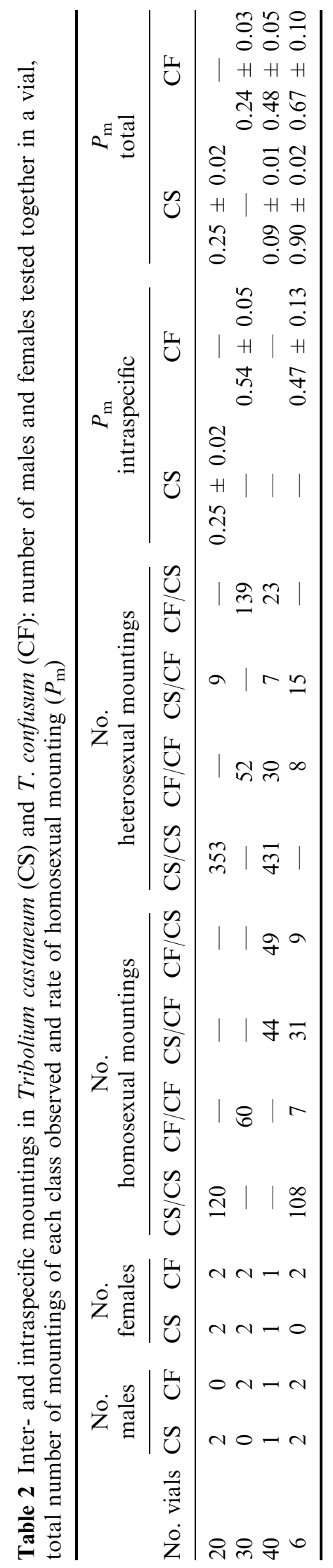

mount, the probability of homosexual mountings given that a mounting takes place $\left(P_{\mathrm{m}}\right)$ can be expressed as

$P_{\mathrm{m}}=\left(\begin{array}{c}m \\ 2\end{array}\right) x /\left[m f y+\left(\begin{array}{c}m \\ 2\end{array}\right) x\right]=1 /\left[1+2 \alpha k m /\left(\begin{array}{ll}m & 1\end{array}\right)\right]$,

where $\alpha$ stands for the probability ratio $y / x$. Therefore, $P_{\mathrm{m}}$ will vary depending both on the experimental conditions under which observations have been made (specified by the values of $m$ and $k$ ) and on the particular properties of the population being tested (defined by the value of the ratio $\alpha$ ). Once the experimental conditions have been defined, $P_{\mathrm{m}}$ can be estimated from the data and the corresponding $\alpha$ can be calculated from the above expression. Ideally, in a contact between males both can mount and be mounted, but in a heterosexual contact only the male can mount. Therefore, with total indiscrimination with respect to sex, $\alpha=0.5$ if both sexes oppose being mounted equally $(x=2 y)$. However, males may resist being mounted more effectively than females $(x<2 y, \alpha>0.5)$, or vice versa $(x>2 y$, $\alpha<0.5)$. Thus, different rates of homosexual mounting may occur, reflecting the balance attained between the male's persistence in mounting and the differential resistance offered by males and females to being mounted. This balance can be experimentally altered and, in CS, the rate of homosexual mounting increased dramatically when highly inbred males were tested together with noninbred females (Serrano et al., 1991). This result can be ascribed to inbreeding, that lowers both male sexual vigour and the resistance to be mounted of males relative to that of vigorous noninbred females.

\section{Results and discussion}

\section{Intraspecific mounting}

The average rate of intraspecific homosexual mounting, together with the total number of mountings recorded during the four observation periods, are shown in Table 1 for each species and experimental situation studied. CS $(m=k=2)$ data from Serrano et al. (1991) were also included for comparison. In CS, the observed rates of homosexual mounting did not significantly differ from previous estimates, both in the Consejo population (Serrano et al., 1991; Castro et al., 1994) and in a recently collected population (Medina, after three generations in the laboratory; Castro, 1988). In CF, however, these rates were much higher than those obtained for CS in the same experimental conditions. In both species, the rate of homosexual mounting was inversely related to the number of females tested, because the probability of a male-female contact increases with that number. The average number of 
mountings (homo- and heterosexual) performed by a male in 30 min was 2.85 in $\mathrm{CS}$ and 0.73 in $\mathrm{CF}$, the former value being similar to that of 3.1 given by Castro et al. (1994).

Within species, no significant differences were found between the values of the probability ratio $\alpha$ calculated in the two experimental situations considered. In CS, $\alpha$ values not significantly different from 0.5 were obtained, in agreement with the hypothesis of the complete lack of sexual discrimination, and both sexes opposing being mounted equally. In $\mathrm{CF}$, however, a much smaller $\alpha$ value of 0.22 was computed. Assuming a lack of sexual discrimination, this $\alpha$ value can be ascribed to a higher resistance of $\mathrm{CF}$ females to being mounted, relative to that of $\mathrm{CF}$ males. However, our observations indicate that $\mathrm{CF}$ males seldom attempted to mount $\mathrm{CF}$ females but, when they did, no female active rejection was perceived. Thus, some mechanism of passive rejection based on a loss of sexual attractiveness of $\mathrm{CF}$ females could also be implied.

Summarizing, the results are compatible with the hypothesis of both species being sexually indiscriminate. However, in the case of $\mathrm{CF}$, a lower sexual attractiveness of females relative to males may also be involved, resulting in a higher rate of homosexual mounting in this species.

\section{Inter- and intraspecific mounting}

The extent to which intraspecific mounting results apply at the interspecific level can also, in principle, be empirically studied by testing together males and females of both species considered. In four different situations (defined by the number and species of the males and females tested together in the same vial), the numbers of mountings observed for all possible combinations (homo- and heterosexual) are given in Table 2, together with the corresponding rates of homosexual mounting, both intraspecific or total (intra- and interspecific).

From the intraspecific data, one could predict that the relative frequencies of different mating pairs will be markedly affected by two factors: (1) the higher sexual activity of CS males relative to CF males, and (2) the lower sexual attractiveness, or the stronger rejection to being mounted, of $\mathrm{CF}$ females relative to CF males. The interspecific data clearly confirmed the influence of both factors: (1) the number of mountings (homo- and heterosexual) performed by CS males was 3-6 times higher than that of CF males, and (2) the number of $\mathrm{CF}$ males mounted was always higher than that of $\mathrm{CF}$ females, irrespective of the mounting male species.

The behaviour of CS and CF males was clearly different. CS males preferentially mounted conspecific individuals (females or males) in a proportion ranging between $71 \%$ and $98 \%$ of all recorded mountings. Thus, when intraspecific homosexual and heterosexual mountings were both feasible (Table 2, row 1), the CS rate of homosexual mounting was similar to that observed at the intraspecific level in comparable experimental conditions $\left(P_{\mathrm{m}}=0.25 ; m=2, k=1\right)$. Of course, that rate was much higher $\left(P_{\mathrm{m}}=0.90\right)$ when heterosexual intraspecific mountings could not be performed (Table 2 , row 4$)$, or much lower $\left(P_{\mathrm{m}}=0.09\right)$ when all homosexual mountings must be interspecific (Table 2, row 3). On the other hand, among CF males, interspecific heterosexual mountings were more frequent than intraspecific ones (Table 2, row 2). Therefore, when CS females were available, the CF total rate of homosexual mounting was lower than that observed at the intraspecific level in analogous experimental conditions. Summarizing, CS males discriminate between species but, in interspecific contacts, preferentially mount CF males. On the contrary, CF males do not discriminate between species.

Both the observed high rates of homosexual mounting in CS and CF (intra- and interspecific) and the different degrees of interspecific discrimination shown by the males of both species, require an evolutionary explanation. Prior to this, some background information on the biology of Tribolium must be given (see Sokoloff, 1974 for a comprehensive review). Both species are rather long-lived, with a mean life expectation of about a year. Mounting (homo- and heterosexual) occurs frequently and is often accompanied by injurious effects to both sexes (Spratt, 1980). Lloyd \& Park (1962) concluded that $\mathrm{CF}$ males are more injurious to their own females than they are to each other, and about equally (but less) injurious to CS males and females. They also found a smaller CS effect, only detected in males. On the other hand, male reproductive success marginally increases with the number of mountings performed per unit of time, as CS and CF show sperm precedence (Lewis \& Austad, 1990; Robinson et al., 1994). In this situation, it may be evolutionarily advantageous for the females of both species to be equally or less sexually attractive than males. This will reduce injuries caused by excessive mounting without practically affecting reproductive potential, as the sperm from a single ejaculation will be enough to fertilize the eggs laid by a female for several weeks (Sokoloff, 1974). On the other hand, CS males are much more sexually active than $\mathrm{CF}$ males and therefore reproductive isolation mechanisms between these species will mostly affect $\mathrm{CF}$ females, as CF males would rarely mount CS females. Thus, the rejection of CF females to being mounted, or their loss of sexual attractiveness, may have been selected as a precopulatory isolation mechanism. 


\section{References}

CASTro, L. 1988. Aspectos genéticos del comportamiento homosexual de Tribolium castaneum. PhD Thesis, Universidad Complutense de Madrid.

CASTRO, L., TORO, M. A. AND LÓPEZ-FANJUL, C. 1994. The genetics properties of homosexual copulation behavior in Tribolium castaneum: artificial selection. Génét. Sél. Évol., 26, 361-367.

FAUSTINI, D. L., BURKHOlder, W. E. AND LAUB, R. J. 1981. Sexually dimorphic setiferous sex patch in the male red flour beetle, Tribolium castaneum (Herbst) (Coleoptera: Tenebrionidae): Site of aggregation pheromone production. J. Chem. Ecol., 7, 465-480.

GRAUR, D. AND wOOL, D. 1985. Unidirectional interspecific mating in Tribolium castaneum and Tribolium confusum: evolutionary and ecological implications. Entomologia exp. appl., 38, 261-265.

JUAN, C. P., VAZQUeZ, P., RUBio, J. M., PeTITPIERRe, E. AND HEWITT, G. M. 1993. Presence of highly repetitive DNA sequences in Tribolium flour beetles. Heredity, 70, 1-8.

LEVINSON, H. Z. AND MORI, K. 1983. Chirality determines pheromone activity in flour beetles. Naturwissenschaften, 70, 190-192.

LEWIS, S. M. AND AUSTAD, S. N. 1990. Sources of intraspecific variation in sperm precedence in red flour beetles. Am. Nat., 135, 351-359.

LlOYD, M. AND PARK, T. 1962. Mortality resulting from interactions between flour beetles in laboratory cultures. Physiol. Zoöl., 35, 330-347.
ROBINSON, T., JOHNSON, N. A. AND WADE, M. J. 1994. Postcopulatory, prezygotic isolation: intraspecific and interspecific sperm precedence in Tribolium spp., flour beetles. Heredity, 73, 155-159.

SERRANO, J. M., CASTRO, L., TORO, M. A. AND LÓPEZ-FANJUL, C. 1991. The genetic properties of homosexual copulation in Tribolium castaneum: diallel analysis. Behav. Genet., 21, 547-558.

SOKoloff, A. 1974. The Biology of Tribolium, vol. 2. Oxford University Press, Oxford.

SPRATT, E.C. 1980. Male homosexual behaviour and other factors influencing adult longevity in Tribolium castaneum (Herbst) and T. confusum (Duval). J. Stored Prod. Res., 16, 109-114.

TAYLOR, C. AND SOKOLOFF, A. 1971. Homo and heterosexual mating of Tribolium in single and mixed species. Tribolium Inf. Bull., 9, 120-121.

THORNHILL, R. AND ALCOCK, J. 1983. The Evolution of Insect Mating Systems. Harvard University Press, Cambridge, MA. UGARKovic, D., PODNAR, M. AND PlOHL, M. 1996. Satellite DNA of the red flour beetle Tribolium castaneum Comparative study of satellites from the genus Tribolium. Mol. Biol. Evol., 13, 1059-1066.

VARDELL, H. H. AND BROWER, J. H. 1976. Sequential interspecific and intraspecific matings between Tribolium castaneum and T. confusum (Coleoptera: Tenebrionidae): effects on fecundity, egg fertility, and number of progeny. Can. Ent., 108, 1391-1394.

WADE, M. J., PATterson, H., ChANG, N. W. AND JOHNSON, N. A. 1994. Postcopulatory, prezygotic isolation in flour beetles. Heredity, 72, 163-167. 